\title{
NUMERICAL INVESTIGATION ON COMPACT MULTIMODE DIELECTRIC RESONATOR ANTENNAS OF VERY HIGH PERMITTIVITY
}

\author{
M. Rotaru, J. K. Sykulski \\ School of ECS, University of Southampton, Highfield, Southampton, SO17 1BJ, UK \\ mr@ecs.soton.ac.uk, jks@soton.ac.uk
}

Keywords: dielectric resonator antenna, antenna design, application of high permittivity material.

\begin{abstract}
Two well known numerical techniques, the Finite Element Method (FEM) and the Finite Integration in Time Domain (FIT), have been applied to study a low volume high permittivity dielectric resonator antenna. The paper demonstrates that the design of a compact size and wide frequency coverage dielectric resonator antenna (DRA) for ISM band is possible by proper selection of the resonator shape in combination with appropriate resonant modes. Numerical results for one particular antenna design are reported.
\end{abstract}

\section{Introduction}

Dielectric resonator antennas (DRAs) have been used in the past due to their excellent characteristics in terms of high radiation efficiency, simple feeding scheme and relatively small size $[2,4]$. While these features can be fully exploited at frequencies of several $\mathrm{GHz}$, or even higher, the DRAs have limited use at lower ISM frequencies (e.g. 902-928 MHz, 2.400-2.500 GHz). Some attempts to design and integrate a DRA of a reasonable size for these frequency bands have been reported [4], but the main drawback of the existent solutions is the size of the antenna and its bandwidth. To obtain a reasonable antenna size in such bands, very high dielectric constants would be required, thus resulting in an unacceptable reduction in bandwidth. As the dielectric constant increases, a rise of the quality factor $(Q)$ of the resonator is observed. In fact $Q$ increases as $\varepsilon^{3 / 2}$ [3] thus resulting in significant reduction of the antenna bandwidth. Different methods have been suggested to improve the bandwidth of high dielectric constant resonator antennas [1]. A combination of two particular approaches appears promising. First, an appropriate resonator shape, that minimizes the surface to volume ratio, is chosen. This is followed by a design of a resonator that can support close resonant modes. The second stage is usually avoided in conventional DRA design since the radiation pattern is strongly dependent on the resonator modes, but in many wireless applications an improved bandwidth is more important than the radiation pattern. Another very important factor in wideband operation is the feeding structure design, such that efficient coupling is obtained for the desired resonant modes.
A novel resonator shape has been developed and is reported here. The simulation results demonstrate that the proposed antenna has a wide bandwidth in the relevant frequency band $(2.4-2.5 \mathrm{GHz})$.

\section{Antenna Structure}

As explained in the introduction, for practical hand held applications at cellular frequencies, a compact DRA is preferred. This implies that high permittivity materials should be used for such antennas. The $Q$ factor of the antenna is the key parameter. It can be directly related to the unloaded $Q$ of the resonant mode excited in the dielectric resonator. The unloaded $Q$ factor can be linked to the geometrical feature of the resonator by the following equation:

$$
Q=2 \omega_{0} \frac{\text { Stored Energy }}{\text { Radiated Power }} \propto 2 \omega_{0} \varepsilon_{r}^{p}\left(\frac{\text { Volume }}{\text { Surface }}\right)^{s}
$$

with $p>_{S} \geq 1$, where $\omega_{0}$ is the resonant angular frequency and $\varepsilon_{\mathrm{r}}$ is the relative dielectric constant. From (1) it is apparent that an increase in $\varepsilon_{\mathrm{r}}$ will result in an increase of $Q$ and this will reduce the antenna bandwidth. Moreover, it may be seen from (1) that - if the volume to surface ratio is minimized - the quality factor will be reduced and the bandwidth of the antenna increased. For example, a rectangular tile-shaped resonator will have a wider bandwidth than a cube-shaped resonator made from same material at the same frequency [1].

In this work we have studied a " $C$ " shaped resonator (Figure 1) which has its thickness much smaller that all the other dimensions.

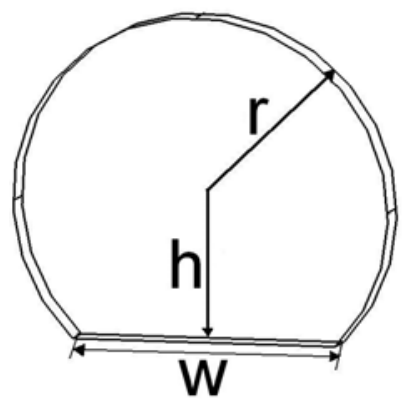

Figure 1. "C" shape resonator

The dimensions of the studied resonator were: $r=14 \mathrm{~mm}$, $h=10 \mathrm{~mm}, w=19.6 \mathrm{~mm}$ and its thickness was $2 \mathrm{~mm}$. Here a material with a dielectric constant of $\varepsilon_{\mathrm{r}}=80$ and loss tangent of 0.0005 at $2 \mathrm{GHz}$ was considered. 
The modes than can be excited into such a resonator have been studied through simulation. A microstrip line feed on a FR4 substrate of $50 \Omega$ characteristic impedance was used to excite this resonator. The flat side of the resonator is placed on top of the microstrip line as sketched in figure 2.

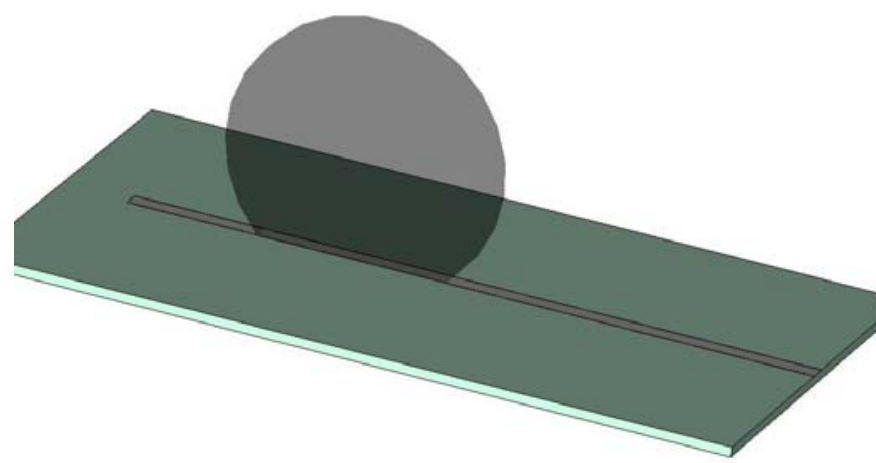

Figure 2. "C" shaped resonator on top of microstrip feed

\section{Results and Discussions}

The above structure was simulated in a full wave solver environment using two different numerical methods. The two methods were used to cross check the results. Both methods gave very similar results that are presented below. The design of such antenna structure would have been very difficult, if not impossible, without a numerical solver. While analytical approximations for finding the resonating modes for rectangular or cylindrical shaped resonators exist [1,3], they are not available for the " $\mathrm{C}$ " shape resonator. The results presented demonstrate that the proposed shape can be used successfully as a radiating element, while it can be fabricated relatively easily from a thin cylinder.

First, the existence of close resonant modes was checked. It has been found that, if the resonator is placed at an optimum distance from the end of the microstrip line, two close modes shown in Figure 3 can be excited. The first mode (Figure $3 a$ ) is excited at $2.49 \mathrm{GHz}$ and the second mode (Figure $3 \mathrm{~b}$ ) at $2.77 \mathrm{GHz}$. These two modes correspond well with the S11 response of the antenna (Figure 4).

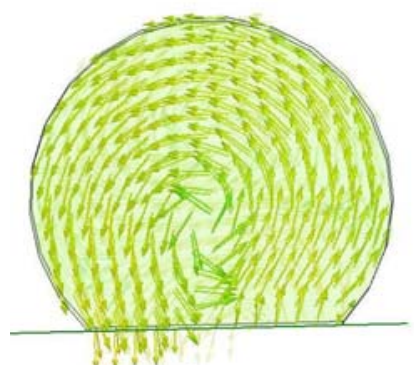

a.

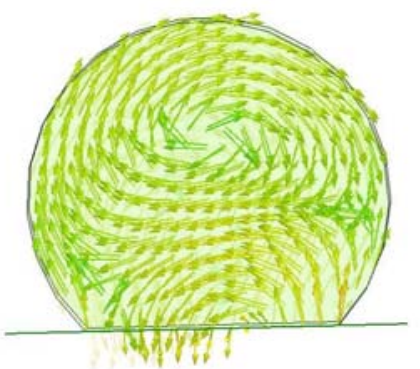

b.
Figure 3. Two resonating modes excited into the " $\mathrm{C}$ " shaped resonator.
The S11 response curve shows that about $25 \%$ of the bandwidth at $-10 \mathrm{~dB}$ level is available due to the reduced quality factor of the resonator and close enough resonating modes. The efficiency of both modes is high (98\% and above) as the losses in the system are very small. The only losses that exist are in fact the microstrip ohmic losses. As expected, the radiation pattern of the two modes is very different but agrees well with the field configuration excited for each mode.

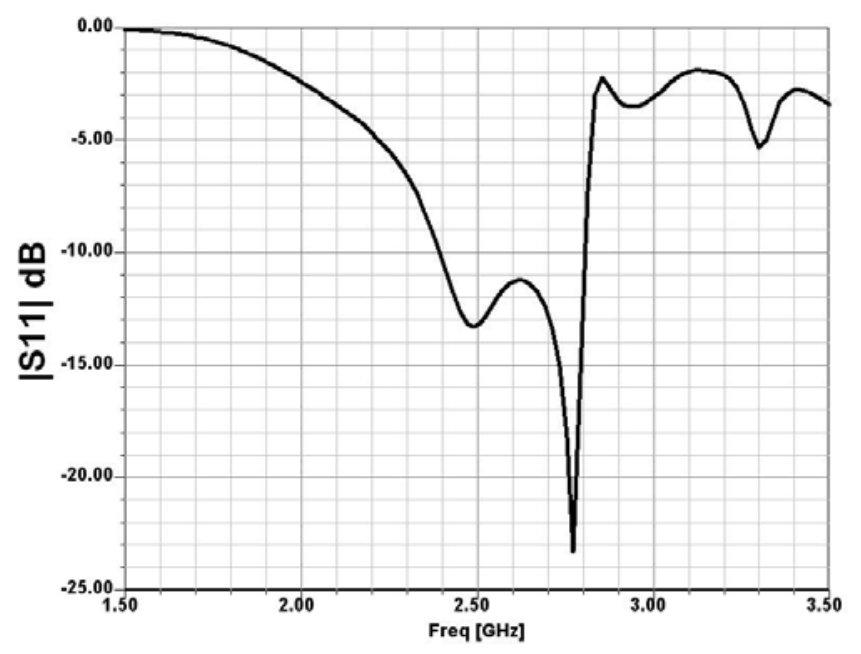

Figure 4. S11 for the thin "C" shaped DRA

\section{Conclusions}

A " $C$ " shaped high permittivity DRA has been studied through numerical modelling. It has been shown that by using a high enough permittivity material, and by selecting an appropriate shape of the resonator coupled with a multimode operation, a compact and wide bandwidth antenna for cellular frequencies could be realised. Further improvement of the size and bandwidth may be possible if higher dielectric constant is used and other shapes that minimize better the volume to surface ratio are employed.

\section{References}

[1] G. Bit-Babik et al. "Multimode Dielectric Resonator Antenna of Very High Permittivity", IEEE Antennas and Propagation Society International Symposium, 2, pp. 1383-1386, (2004).

[2] S. A. Long et al. "The resonant cylindrical dielectric cavity antenna", IEEE Trans. on Antenna and Propagation, 31(3), pp. 406-412, (1983).

[3] R. K. Mongia et al. "Theoretical and Experimental Investigation of Rectangular Dielectric Resonator Antennas", IEEE Trans. on Antenna and Propagation, 45(9), pp. 1348-1356, (1997).

[4] Yuan Gao et al. "A Compact Wide Band Hybrid Dielectric Resonator Antenna", IEEE Microwave and Wireless Components Letters, 16(4), pp. 227229, (2006). 